\title{
Auto Inter-Carrier Frequency Offset Rectification Using Conjugate Cancellation in Multi-carrier CDMA Broadband Communications
}

\author{
Mohamed Khedr $^{* *}$, Entesar Gemeay ${ }^{*}$, Said El-noubi ${ }^{\dagger}$ and Mohamed Nasr ${ }^{*}$
}

\begin{abstract}
Self cancellation schemes confirmed its effectiveness for mitigating the intercarrier interference (ICI) in orthogonal frequency multiplexing (OFDM) system. The main source of ICI is the carrier frequency offsets (CFO) in mobile radio channel which destroys the carrier's orthogonality. In this paper, we employ a self cancellation scheme known as the conjugate self cancellation scheme to reduce the impact the ICI in Multicarrier CDMA systems that use Time-Frequency domain (TF or Hybrid) spreading and are used in broadband wireless communications. We show with proof that TF-domain spreading Multicarrier CDMA system with conjugate cancellation $(\mathrm{CC})$ works significantly better than conventional MC-CDMA system if the normalized frequency offset is less than 0.3 in the fading channels and in additive white Gaussian channel. The $\mathrm{CC}$ algorithm provides a high carrier to interference power ratio (CIR) in the small frequency offset compared with other self cancellation schemes and uses low cost receiver without increase in the system complexity. The drawback of this algorithm is the reduction in bandwidth efficiency, which can be compensated by using larger signal alphabet sizes. Simulation results show good agreement with the numerical results.
\end{abstract}

Index Terms-multicarrier CDMA, carrier frequency offset, frequency-domain spreading, time-domain spreading, Nakagami fading channel, conjugate cancellation.

\section{INTRODUCTION}

Multicarrier communication techniques [1-3] are recognized as potential candidates for current and future broadband wireless systems $[4,5]$. This is mainly due to their attractive features in terms of robustness against channel distortions and narrowband interference, high spectral efficiency, high flexibility in resource management and ability to support adaptive modulation/coding schemes. However, multicarrier systems are very sensitive to phase noise (PHN) and carrier frequency offset (CFO) caused by Doppler shift or by the oscillator instabilities [6-8]. The CFO in MC-DS-CDMA not only makes channels time-varying, but also destroys the orthogonality among sub-carriers, which causes intercarrier interference (ICI) and multi-access interference (MAI) [9]. Even if orthogonal spreading codes are used, MC-DS-CDMA with CFO still suffers from MAI and ICI. However, there are several CFO suppression

\footnotetext{
**Electronics and Communication Department, Arab Academy for Science and Technology,

*Communications Engineering, Tanta University,

$\uparrow$ Electrical Engineering, Alexandria University
}

schemes were proposed in literature [10-12]. Among these schemes is the ICI self-cancellation or polynomial cancellation coding [10] scheme. Self-cancellation methods have received much attention due to its simplicity without increasing the system cost. In this paper, we concentrate on the conjugate cancellation (CC) scheme [11] which has architecture that resemble the idea of Alamouti scheme [11], but with a simple, low cost implementation and without channel estimation requirements. In this scheme, two-path algorithm is developed for combating ICI. The first path employs the regular TF-domain spread MC DS-CDMA system. The second path employs conjugate transmission at the transmitter, and a conjugate cancellation (CC) scheme for mitigating ICI of TF-domain spread MC DS-CDMA system at the receiver. The proposed multicarrier system in this paper is the so called Time-Frequency domain (TF-domain or Hybrid) spreading MC DS-CDMA system [13]. The benefits of using the F-domain spreading [1] instead of the F-domain repetition as in the conventional MC DS-CDMA is to mitigate the deficiency that the number of users decreases upon increasing the F-domain repetition depth. Thus, the total number of users supported by TF-domain spread MC DS-CDMA is determined by the product of the T-domain spreading factor and the F-domain spreading factor. In addition, the TF-domain spreading MC DS-CDMA system exhibits robusteness against the impairments of wireless channels in comparison with MC-CDMA system [9], as shown in this paper. The aim of this paper is to investigate the effect of using the CC scheme [11] to mitigate the ICI in the TF-domain spread MC DS-CDMA system, and evaluate the system performance over the Nakagami-m fading channels. Nakagami distribution provides a more general and versatile way to model the wireless channels [4],[14], and has gained a widespread application in the modeling of the wireless fading channels. The conditional probability of the Bit-Error Rate (BER) of the TF-domain spread MC DS-CDMA system is evaluated over the Nakagami- $m$ fading channels by using the Standard Gaussian approximation (SGA) approach [15]. This approach is more accurate and much simpler at low signal-to-noise ratio (SNR) than other techniques. The performance of the TF-domain spread MC DS-CDMA is compared with that obtained in [9] for MC-CDMA system which is a promising candidate multiple access scheme for next generation of broadband wireless technology [4].

This paper is organized as follows, the proposed system model for TF-domain spread MC DS-CDMA system with $\mathrm{CC}$ scheme is illustrated in section II. The analysis and 
derivation of the BER for TF-domain spread MC DS-CDMA system is presented in section III. Section IV, shows the numerical and simulation results. Finally, the conclusion is presented in section $\mathrm{V}$.

\section{SYSTEM MODEL}

\section{A. Transmitter Model}

The transmitter block diagram of the TF-domain spread MC DS-CDMA system with the CC scheme is shown in Fig. 1 for a single user. The binary data symbols $b_{k}$ of the $k t h$ user is first spread in time domain using the random signature sequence $a_{k}(t)$. The spread signal is divided into $M$ parallel branches, where $M$ refers to the length of frequency spreading. Then, each branch of the signal is multiplied by the corresponding chip value of the $F$-domain spreading sequence $F_{\mathrm{k}}$. Following, the $F$-domain spreading, each of the $M$ branch signals modulates one of the $M$ subcarriers frequencies using Inverse Fast Fourier Transform IFFT block. We assume that the number of subcarriers is equal to the frequency spreading factor $M$. The $m^{\text {th }}$ IFFT output in the $i^{\text {th }}$ bit interval $\left[0, T_{b}\right)$ can be expressed as

$$
v_{k, n}=\sqrt{A} \sum_{h=0}^{M-1} b_{k}^{i} a_{k}(t) F_{k, n} \frac{1}{M} e^{\frac{j 2 \pi}{M} n h} \quad n=0, \ldots, M-1 .
$$

where $A$ is the transmitted signal power. The time-frequency spreading codes are assumed independent random codes. The time-domain spreading waveform of the $k^{\text {th }}$ user is given as

$$
a_{k}(t)=\sum_{l=0}^{\infty} a_{k, l} P\left(t-l T_{c}\right)
$$

where $P(t)$ represents the rectangular T-domain chip waveform in chip interval $\left[0, T_{c}\right)$, and $a_{k, l} \in\{+1,-1\}$ is generated at the rate of $N$ chips. Assuming the transmitted power of each user is identical and equal to $A$. The transmitted signal of the first path or the regular signal is given as

$$
S_{k}(t)=\sqrt{A} \sum_{n=-N_{g}}^{M-1} \sum_{h=0}^{M-1} b_{k}^{i} a_{k}(t) F_{k, n} \frac{1}{M} e^{\frac{j 2 \pi}{M} n h}
$$

and the signal for the second path or conjugate signal is given as

$$
\hat{S}_{k}(t)=\sqrt{A} \sum_{n=0}^{M-1} \sum_{h=0}^{M-1} b_{k}^{i} a_{k}(t) F_{k, n} \frac{1}{M} e^{\frac{-j 2 \pi}{M} n h}
$$

The two paths are transmitted using the time-division multiplexing (TDM).

\section{B. Receiver Model}

After the insertion of guard interval, the $S_{k}(t)$ signal is transmitted through a fading channel. The channel that will be considered in this analysis is the Nakagami-m fading channel [14]. This fading distribution has gained a lot of attention lately as Nakagami- $m$ distribution often gives the best fit to land-mobile and indoor mobile multipath propagation. The parameter $m$ in Nakagami distribution characterizes the severity of the fading channel, where $m=1$ represents Rayleigh fading, $m>1$ can be approximated the Rician and lognormal distributions, while $m \rightarrow \infty$ corresponds to the Gaussian distribution. With the proper selection of the number of subcarriers, we can consider that, each subcarrier experiences independent flat fading. Assuming, there are $\mathrm{K}$ asynchronous TF-domain spread MC DS-CDMA users in the system with the same transmitted power and with perfect power control, the received signal during the $i^{\text {th }}$ bit interval, impaired by the frequency offset is given as

$$
\begin{gathered}
r(t)=\sqrt{A} \sum_{k=1}^{K} \sum_{n=-N_{g}}^{M-1} \sum_{h=0}^{M-1} \alpha_{k, n} b_{k}^{i}\left(t-\tau_{k}\right) a_{k}\left(t-\tau_{k}\right) F_{k, n} \\
\frac{1}{M} e^{\frac{j 2 \pi}{M}(n+\mathcal{E}-g) h} \cdot e^{-j \psi_{k, n}}+\eta(t)
\end{gathered}
$$

where the channel phases $\psi_{k, n}$ are i.i.d. random variable having uniform distribution in $[0,2 \pi), \tau_{k}$ is the propagation delay for the $k^{\text {th }}$ user, $\mathcal{E}$ is the normalized frequency offset, $\eta(t)$ is additive white Gaussian noise (AWGN) with a double-sided power spectral density of value $\left(N_{0} / 2\right)$ [16], while the fading amplitudes $\alpha_{k, n}$ are independent Nakagami random variables with a probability density function given by [14]

$$
p\left(\alpha_{k, n}, \Omega_{k, n}\right)=\frac{2 \alpha_{k, n}^{2 m-1}}{\Gamma(m)}\left(\frac{m}{\Omega_{k, n}}\right)^{m} \exp \left(\frac{-m \alpha_{k, n}^{2}}{\Omega_{k, n}}\right)
$$

where $\Omega_{k, n}=\mathrm{E}\left(\alpha_{k, n}^{2}\right), \mathrm{E}[$.$] refers to the expectation$ operator, $\Gamma($.$) is the Gamma function, and m$ is the Nakagami fading parameter, which is equal to $m=\mathrm{E}^{2}\left[\alpha_{k, n}^{2}\right] / \operatorname{var}\left[\alpha_{k, n}^{2}\right]$.

After removing the guard interval from the receiving signal the received signal at the receiver side is the combination between the first path and the conjugate of the second path, thus after the FFT process, the signal at the subcarrier $g$ can be expressed as

$$
\begin{gathered}
y_{g}=\sqrt{A} \sum_{n=0}^{M-1} \alpha_{d, n} b_{d}^{i}(t-\tau) a_{d}(t-\tau) F_{d, n}\{S(n-g)+\widehat{S}(n-g)\} \\
+\sum_{n=0}^{M-1} \sum_{\substack{k=1 \\
k \neq d}}^{K} \alpha_{k, n} e^{-j \psi_{k, n}} b_{k}^{i}\left(t-\tau_{k}\right) a_{k}\left(t-\tau_{k}\right) F_{k, n} . \\
\{S(n-g)+\hat{S}(n-g)\}+\eta_{g}
\end{gathered}
$$

The weighting function $S(n-g)$ is given as

$$
\begin{aligned}
& S(n-g)=\frac{1}{M} \sum_{h=0}^{M-1} e^{\frac{j 2 \pi}{M}(n-g+\mathcal{E}) h} \\
& =\frac{\sin (\pi(n-g+\varepsilon))}{M \sin \left(\frac{\pi}{M}(n-g+\varepsilon)\right)} e^{\left(j\left\{\pi\left(1-\frac{1}{M}\right)(n-g+\varepsilon)\right\}\right)}
\end{aligned}
$$

and the conjugate weighting function $\widehat{S}(n-g)$ is given as 


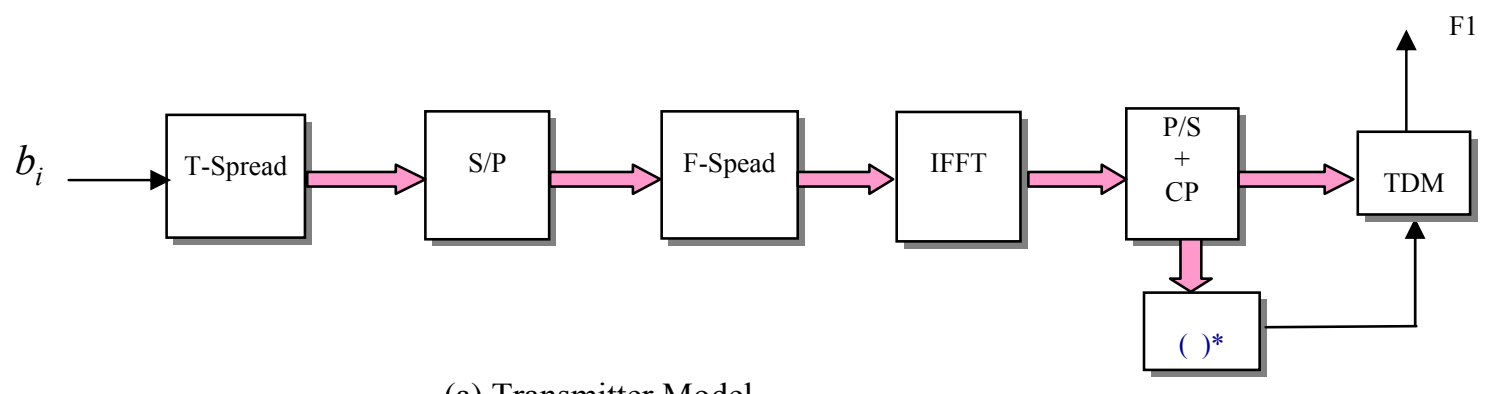

(a) Transmitter Model

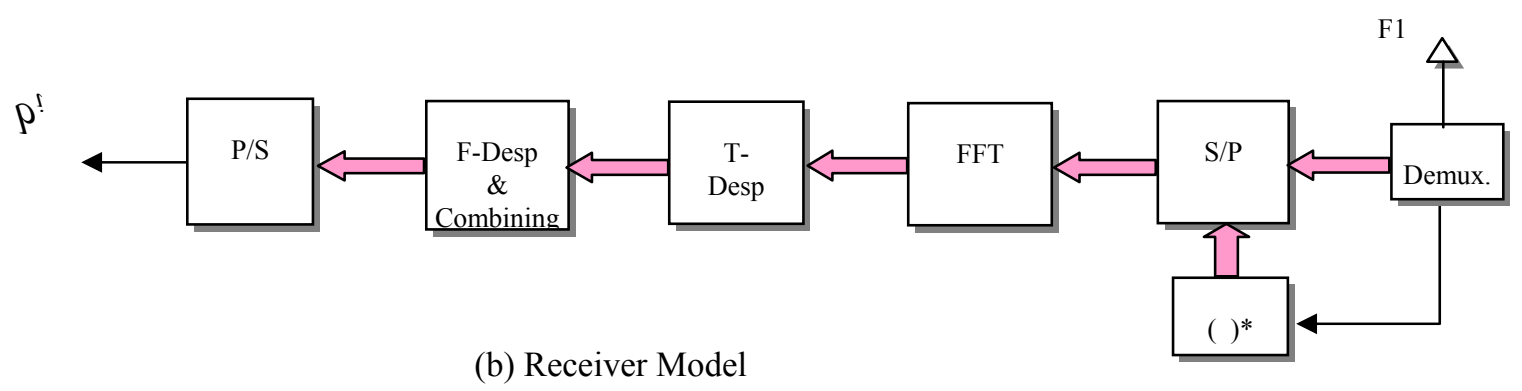

Fig.1 Block Diagram of TF-domain spread MC DS-CDMA system Using Conjugate cancellation scheme

$$
\begin{aligned}
& \widehat{S}(n-g)=\frac{1}{M} \sum_{h=0}^{M-1} e^{\frac{j 2 \pi}{M}(n-g-\varepsilon) h} \\
& =\frac{\sin (\pi(n-g-\varepsilon))}{M \sin \left(\frac{\pi}{M}(n-g-\varepsilon)\right)} e^{\left(j\left\{\pi\left(1-\frac{1}{M}\right)(n-g-\varepsilon)\right\}\right)}
\end{aligned}
$$

It can be seen that, the weighting function in (8) is similar to that in (9), but the sign of the frequency offset term, $\mathcal{E}$ is changed from positive to negative. Therefore, when the frequency offset $\mathcal{E}>0$, it will result in a shift to the right operation on the weighting function of (9) as opposed to a shift to the left of (8).

It can be said that with the using of the $\mathrm{CC}$ scheme, we will obtain a new weighting function $W(n-g)$ which is given as

$$
W(n-g)=S(n-g)+\widehat{S}(n-g) \text {. }
$$

The weighting function in (10) is compared with the regular weighting function in (8) as shown in Fig. 2. As illustrated in Fig.2a and 2b, the new weighting function gives gain to the desired signal nearly twice to that of the regular value at low frequency offset $(\varepsilon<0.2)$. But, at high frequency offset ( $\varepsilon>0.3$ ) the regular system performs better than the $\mathrm{CC}$ system.

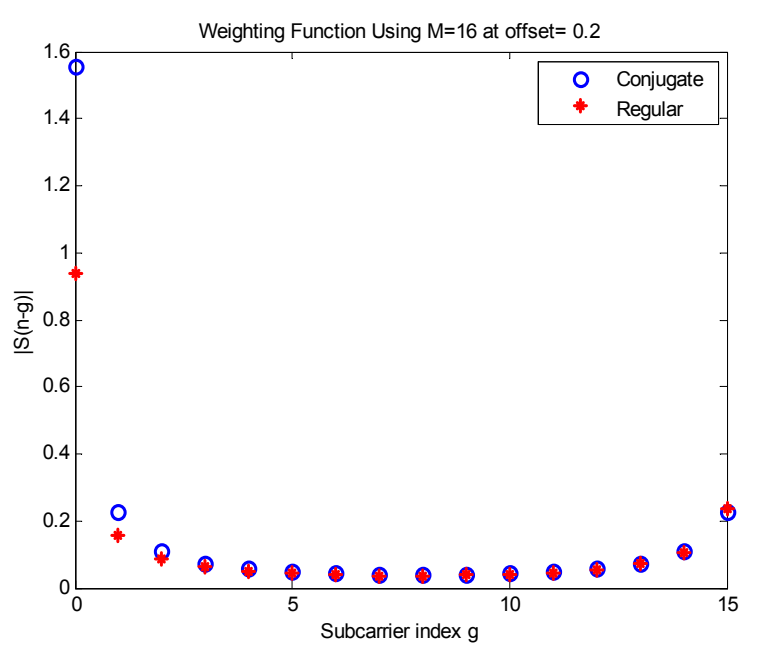

Fig. 2a weighting function at $\varepsilon=0.2$, for regular and conjugate scheme .

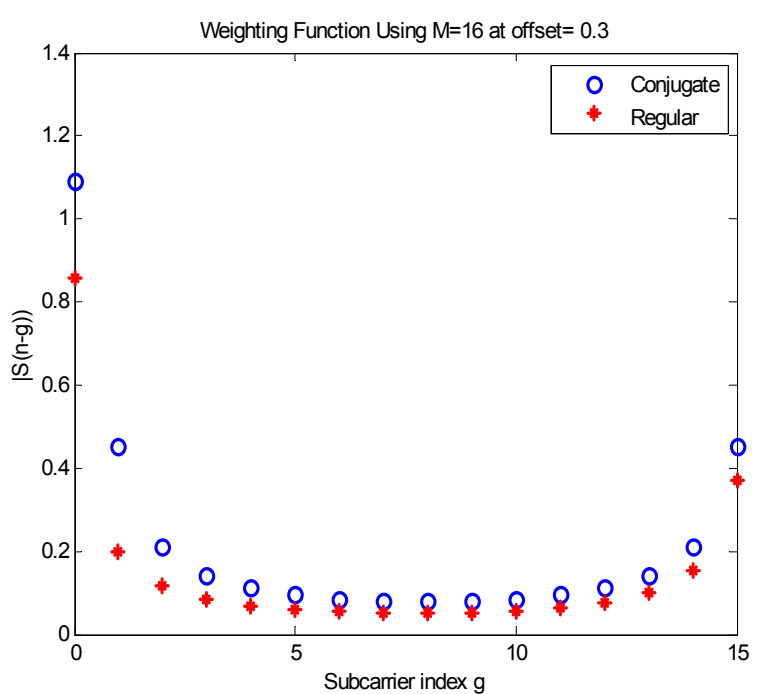

Fig. $2 \mathrm{~b}$ Weighting function at $\varepsilon=0.3$, for regular and conjugate scheme 
After time and frequency despreading using the codes of the desired user and maximum ratio combining (MRC), the decision signal is given as

$$
\begin{gathered}
Z_{d}=\sqrt{A} \sum_{g=0}^{M-1} \alpha_{d, g}^{2} F_{d, g}^{2} b_{d}^{i} W(0)+\sqrt{A} \sum_{g=0}^{M-1} \sum_{\substack{n=0 \\
n \neq g}}^{M-1} \alpha_{d, g} . \\
\alpha_{d, n} b_{d}^{i} F_{d, n} F_{d, g} \cdot W(n-g)+\sqrt{A} \sum_{g=0}^{M-1} \sum_{\substack{n=0 \\
n \neq g}}^{M-1} \sum_{\substack{k=1 \\
k \neq d}}^{K} \alpha_{d, g} . \\
\alpha_{k, n} \Re_{k} F_{d, g} F_{k, n} \cdot W(n-g) \cdot e^{-j \psi_{k, n}}+\sqrt{A} \sum_{\substack { g=0 \\
\begin{subarray}{c}{k=1 \\
k \neq d{ g = 0 \\
\begin{subarray} { c } { k = 1 \\
k \neq d } }\end{subarray}}^{K-1} \alpha_{d, g} \alpha_{k, g} . \\
\mathfrak{R}_{k} F_{d, g} F_{k, g} \cdot e^{-j \psi_{k, g}} \cdot W(0)+\sum_{g=0}^{M-1} \alpha_{d, g} F_{d, g} \eta_{d, g} .
\end{gathered}
$$

where $\mathfrak{R}_{k}$ in (9) is given as in [17]

$$
\mathfrak{R}_{k}=\left[b_{k}[-1] R_{k}\left(\tau_{k}\right)+b_{k}[0] \hat{R}_{k}\left(\tau_{k}\right)\right]
$$

where $R_{k}, \hat{R}_{k}$ are the cross-correlation between the $\mathrm{T}$-domain spreading sequences of the $d t h$ user and $k t h$ user, which are given as

$$
\begin{aligned}
& R_{k}\left(\tau_{k}\right)=\int_{n T_{c}}^{n T_{c}+\tau_{k}} a_{d}(t) a_{k}\left(t-\tau_{k}\right) d t \\
& \hat{R}_{k}\left(\tau_{k}\right)=\int_{n T_{c}+\tau_{k}}^{(n+1) T_{c}} a_{d}(t) a_{k}\left(t-\tau_{k}\right) d t
\end{aligned}
$$

The above equation can be expressed as

$$
Z_{d}=D_{d}+I_{s}+I_{I C I}+I_{M A I}+N_{d}
$$

where the first term in (11) $D_{d}$ is the desired symbol of the $d^{\text {th }}$ user, the second term $\left(I_{s}\right)$ is the self interference, the third term $\left(I_{I C I}\right)$ is the intercarrier interference which is due to the interference, the fourth term $\left(I_{M A I}\right)$ is multiple access interference, and the final term $\left(N_{d}\right)$ is the AWGN noise signal.

\section{BIT ERROR RATE ANALYSIS}

In this section, we derive the downlink BER of the TF-domain spread MC DS-CDMA system over Nakagami-m fading channel and in the presence of carrier frequency offset. The channel fading and phase shift variables are assumed to be constant within the symbol-duration. Based on the Gaussian approximation [15], the decision variable $Z_{d}$ in (11) can be approximated as a Gaussian random variable having a mean given by

$$
\begin{aligned}
\mathrm{E}\left[Z_{d}\right] & =\mathrm{E}\left[\sqrt{A} \sum_{g=0}^{M-1} \frac{\alpha_{d, g}^{2}}{M} b_{d}^{i} \sum_{h=0}^{M-1} \frac{1}{M} e^{\frac{j 2 \pi}{M} \varepsilon h}\right] \\
& =\sqrt{A} \sum_{g=0}^{M-1} \frac{\mathrm{E}\left(\alpha_{d, g}^{2}\right)}{M} b_{d}^{i} S_{0}
\end{aligned}
$$

where as stated in [17], we assume a negative exponentially decaying multipath intensity profile (MIP) distribution given by $\Omega=\mathrm{E}\left(\alpha_{k, n}^{2}\right)=\Omega_{0} \exp (-\zeta l), \zeta \geq 0, l$ is tap number, $\Omega_{0}$ is the average signal strength corresponding to the first resolvable path and $\zeta$ is the rate of average power decay. We assume that all the subcarrier signals of the different users have the same MIP distribution. The variance of self interference can be calculated as follows

$$
I_{s}=\sum_{g=0}^{M-1} \alpha_{d, g} I_{g}^{s}
$$

where the variance of $I_{g}^{s}$ is given as

$$
\begin{array}{r}
\operatorname{var}\left(I_{g}^{s}\right)=\mathrm{E}\left[\left(\sqrt{A} \sum_{\substack{n=0 \\
n \neq g}}^{M-1} \sum_{h=0}^{M-1} \alpha_{d, n} F_{d, g} F_{d, n} \cdot W(n-g)\right.\right. \\
=\frac{A}{M^{2}} \sum_{\substack{n=0 \\
n \neq g}}^{M-1} \mathrm{E}\left(\alpha_{d, n}^{2}\right) \cdot W(n-g)^{2}=\frac{A \Omega}{M^{2}} \sum_{\substack{n=0 \\
n \neq g}}^{M-1} W(n-g)^{2}
\end{array}
$$

By following the same steps above, we can evaluate the variance of both intercarrier interference and multiple access interference, which are given as

$$
\operatorname{Var}\left[I_{I C I}\right]=\frac{A(K-1) \Omega}{3 N M^{2}} \sum_{n=1}^{M-1} W(n)^{2} \sum_{g=0}^{M-1} \alpha_{d, g}^{2}
$$

and

$$
\operatorname{Var}\left[I_{M A I}\right]=\frac{A(K-1) \Omega}{3 N M^{2}} \cdot W(0)^{2} \cdot \sum_{g=0}^{M-1} \alpha_{d, g}^{2}
$$

since $\mathrm{E}\left[\Re_{k}^{2}\right]=(2 / 3 N)$ and $\mathrm{E}\left[\operatorname{Real}\left(e^{-j \psi_{k, n}}\right)^{2}\right]=(1 / 2)$ as given in [17]. Finally, the variance of the complex Gaussian random process shown in (11) can be obtained as follows, the variance of the noise term $N_{d}$ is given by

$$
\operatorname{Var}\left[N_{d}\right]=\frac{\left(N_{0}\right)}{M} \sum_{g=0}^{M-1} \alpha_{d, g}^{2}
$$

then from (19) to (22), we can obtained the variance of the decision variable $Z_{d}$, which can be written as

$$
\begin{aligned}
\operatorname{Var}\left[Z_{d}\right]= & A\left[\left(\frac{1}{M^{2}}+\frac{(K-1)}{3 N M^{2}}\right) \sum_{n=1}^{M-1} W(n)^{2}+\frac{(K-1)}{3 N M^{2}} \cdot W(0)^{2}\right. \\
& \left.+\frac{\left(N_{0} / A\right)}{M \Omega}\right] \cdot \sum_{g=0}^{M-1} \alpha_{d, g}^{2}
\end{aligned}
$$

From the mean and the variance shown in (13) and (19) respectively, we can express the average BER of the TF-domain spread MC DS-CDMA system using the CC scheme over Nakagami-m fading channel as

$$
P_{b}(\gamma)=Q\left(\sqrt{\frac{\left(\mathrm{E}\left[Z_{d}\right]\right)^{2}}{\operatorname{Var}\left[Z_{d}\right]}}\right)=Q\left(\sqrt{\gamma_{d} \sum_{g=0}^{M-1} \frac{\alpha_{d, g}^{2}}{\Omega}}\right)
$$

where

$$
\gamma_{d}=\frac{|W(0)|^{2}}{\left[\left(\Omega_{0} \frac{\left(E_{b} / N_{0}\right)}{M}\right)^{-1}+\left(1+\frac{K-1}{3 N}\right) \sum_{n=1}^{M-1}|W(n)|^{2}+\left(\frac{K-1}{3 N}\right)|W(0)|^{2}\right]}
$$

for BPSK signal modulation $E_{b}$ is the energy during the 
bit interval. The Gaussian Q-function can be represented in the form of $Q(x)=(1 / \sqrt{2 \pi}) \int_{x}^{\infty} e^{-\left(t^{2} / 2\right)} d t$ as in [16], but for a convenient analytical expression of the average BER, we use the alternative expression of the Q-function, which was presented in [8] and is given as

$$
Q(x)=\frac{1}{\pi} \int_{0}^{\pi / 2} \exp \left(-\frac{x^{2}}{2 \sin ^{2} \theta}\right) d \theta \quad x \geq 0
$$

To compute the average BER, we must statistically average (24) over the joint probability density function of fading attenuations $p_{\alpha}\left(\alpha_{d, 0}, \ldots, \alpha_{d, M-1}\right)$. For the assumption of independent fading channels at different subcarriers, the average BER can be expressed as

$$
P_{b}=\frac{1}{\pi} \int_{0}^{\pi / 2}\left(\frac{m \sin ^{2} \theta}{\gamma_{d}+m \sin ^{2} \theta}\right)^{m M} d \theta
$$

The transmitted power of each path of the system with the CC scheme is half of that of the regular TF-MC DS-CDMA. The BER in AWGN is obtained from equation (11) by setting $\Omega=1$. Thus the probability of BER is given as

$$
P_{b}(\gamma)=Q\left(\sqrt{\frac{\left(\mathrm{E}\left[Z_{d}\right]\right)^{2}}{\operatorname{Var}\left[Z_{d}\right]}}\right)=Q(\sqrt{\lambda})
$$

where $\lambda$ is given as

$$
\lambda=\frac{|W(0)|^{2}}{\left[\left(E_{b} / N_{0}\right)^{-1}+\left(\frac{1}{M}+\frac{K-1}{N M}\right) \sum_{n=1}^{M-1}|W(n)|^{2}+\left(\frac{K-1}{N M}\right)|W(0)|^{2}\right]}
$$

Next, we investigate the performance of the proposed system based on the probability of bit error rate shown in (22).

\section{PERformance Results}

In this section, we evaluate the performance of TF-domain spread MC DS-CDMA system with the CC scheme. In the following, we assume random time and frequency spreading codes. Fig. 3 compares the simulation results of TF-domain spread MC DS-CDMA system with the theoretical performance shown in (22) in a Rayliegh fading channel $(\mathrm{m}=1)$, using random spreading sequences at different values of frequency offset. The simulation results are performed by using MATLAB with a 500 iteration, BPSK signal with $100 \mathrm{~ms}$ length, and 10 us bit duration. The number of users in the simulation is three users with a random time and frequency spreading codes for each user. The COST 207 [11] standard urban channel is used in the simulation to model the fading channel. We can observe that, the simulation results agree with the numerical results, but there are insignificant differences at high Eb/N0. The simulation simulation and numerical results of the TF-MC DS-CDMA system in AWGN channel is shown in Fig. 4 using $N=16$ chips, $M=8$ subcarriers, and

$\mathrm{K}=3$ users.

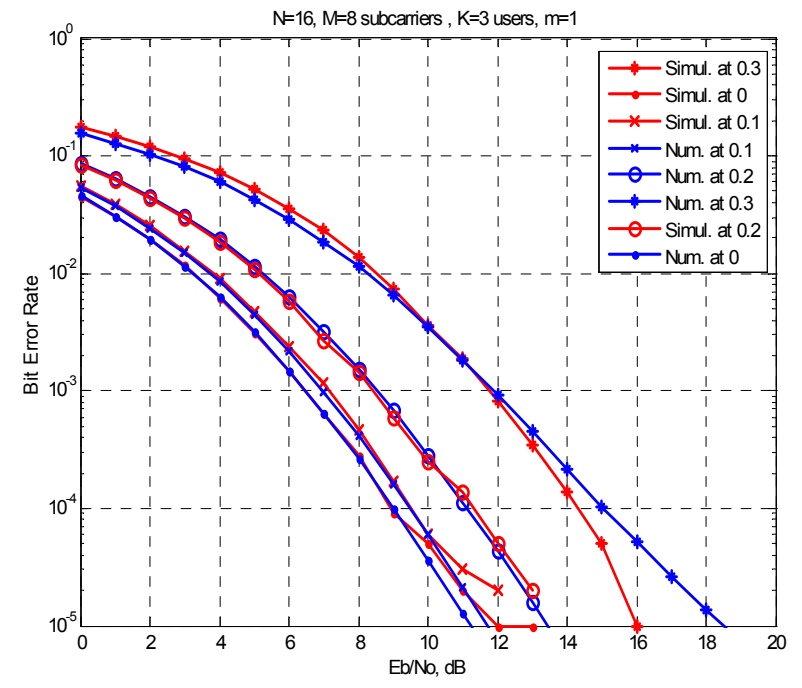

Fig. 3 BER of TF-domain spread MC DS-CDMA system using conjugate cancellation in multipath Rayleigh fading channel

In Fig.5, we compare the performance of the system that uses the CC scheme with that system without CC scheme at $\varepsilon$ $=0.1$ and 0.2 . From the results in Fig. 5 we notice that, at $\varepsilon=$ 0.1 , and at the probability of $\mathrm{BER}=10^{-4}$, the system without CC scheme needs $4 \mathrm{~dB}$, more than the system with $\mathrm{CC}$ scheme to achieve the same performance, while at $\varepsilon=0.2$ needs $6 \mathrm{~dB}$. So, we can say that the system with $\mathrm{CC}$ scheme gives a power gain with respect to the regular system. The BER performance of the proposed system versus the number of users is shown in Fig.6. We observe that the system with the $\mathrm{CC}$ has a high capacity compared with the regular system at different values of frequency offset. At $\varepsilon=0, \mathrm{BER}=10^{-5}$ the system with the $\mathrm{CC}$ can achieve double the capacity than the system without $\mathrm{CC}$, while at $\varepsilon=0.1$, and at the same BER the system with $\mathrm{CC}$ achieves a superior capacity (nearly 5 times) than the regular system. The effect of the number of subcarriers on the performance of the system is shown in Fig.7. By increasing the number of the subcarriers in the system with the $\mathrm{CC}$ scheme at high frequency offset ( $\varepsilon=$ 0.3 ), the performance of the system improves when the frequency offset and at $E_{b} / N_{o}=10 d B \cdot E_{b} / N_{o}>12 d B$, but we notice that when the number of subcarrier exceeds 64 $(M>64)$, the curves convergence and the performance does not improve more this value. In Fig.8, we compare between the TF-MC DS-CDMA system with and without CC scheme and the conventional MC-CDMA system versus the normalized frequency offset and at $E_{b} / N_{o}=10 \mathrm{~dB}$. The results in Fig. 8 show that the TF-MC DS-CDMA system with the $\mathrm{CC}$ has a superior performance over the system without the $\mathrm{CC}$ and over the conventional MC-CDMA system until $\varepsilon$ $=0.32$, above this value the regular system (without the $\mathrm{CC}$ ) has a better performance than it. Fig.9 illustrates the effect of the channel fading parameter $m$ on the performance of the system in the case of using the CC scheme and in the regular system at different values of the CFO, and at $E_{b} / N_{o}=12 d B$. As we notice from the figure, the performance with the CC scheme is improved more than that for the regular system, and as expected the BER improves as the amount of the fading parameter $m$ increases for both 
system. The performance of both system in Fig.9 with the non-fading channel can be obtained until $m$ parameter approaches 10 .

\section{CONCLUSION}

In this paper, we employed the Conjugate self cancellation scheme to mitigate the ICI for a Multicarrier CDMA system which uses a Time-Frequency (TF or a hybrid) spreading codes. We evaluated the conditional probability of BER of the TF-domain spread MC DS-CDMA system which uses the CC scheme over AWGN and Nakagami-m fading channels by using the SGA algorithm. The TF-MC DS-CDMA with conjugate cancellation (CC) works significantly better than a regular system (without $\mathrm{CC}$ scheme) if the normalized frequency offset is less than 0.3 in the additive white Gaussian noise (AWGN) and in the fading channels. The simulation results showed a good agreement with the numerical results in AWGN channel and in the frequency-selective-fading channel. The capacity of the system with the CC is increased to double than in the regular system. In addition to that, the TF-domain spread MC DS-CDMA system outperforms the conventional MC-CDMA system which is a promising candidate for next generation of wireless technology. The drawback of most self cancellation schemes are the reduction in the bandwidth efficiency, which can be compensated by using larger signal alphabet sizes. From the analysis and the simulation results of the TF-domain spread MC DS-CDMA system, we can say that this system can achieve the requirements of the next broadband wireless technology.

\section{REFERENCES}

[1] L.L. Yang, and L.Hanzo, "Multicarrier DS-CDMA: A Multiple Access Scheme for Ubiquitous Broadband Wireless Communications," IEEE Commun.Mag., Oct. 2003, pp. 116-124.

[2] Z.Wang and G. B. Giannakis, "Wireless multicarrier communications," IEEE Signal Processing Magazine, vol. 17, no. 3, pp.29-48, 2000.

[3] S. Hara, and R. Prasad, "Overview of Multicarrier CDMA," IEEE Commun. Mag., Dec. 1997, pp. 126-133.

[4] V. Tarokh, New Directions in Wireless Communications Research, New York, Springer, 2009.

[5] M. L. Roberts, M. A. Temple, R. F. Mils, and R. A. Raines, " Evolution of The Air Interface of Cellular Communications Systems Toward 4G Realization, “IEEE Commun. Surveys \& Tutorials, $1^{\text {st }}$ Quarter 2006.

[6] J. Jang and K. B. Lee, "Effects of frequency offset on MC/CDMA system performance," IEEE Communications Letters, vol. 3, no.7, pp. 196-198, 1999

[7] H. Steendam, and M. Moeneclaey, "The Effect of Carrier Frequency Offsets on Downlink and Uplink MC-DS-CDMA, " IEEE J. Select. Areas Commun., Vol. 19, No. 12, Dec. 2001, pp. 2528-2536.

[8] H. Steendam, H. Bruneel, and M. Moeneclaey, "A Comparison Between Uplink and Downlink MC-DS-CDMA Sensitivity to Static Timing and Clock Frequency Offsets," IEEE Trans. Signal Proc., Vol. 53 , No. 10 , ct. 2005 , pp. 3869-38.

[9] W.M. Jang, L. Nguyen, and P. Bidarkar, " MAI and ICI of Synchronous Downlink MC-CDMA with Frequency Offset, " IEEE Trans. Wireless Commun. Vol. 5, No. 3, Mar. 2006, pp. 693-703.

[10] Y. Zhao and S. Haggman, "Intercarrier Interference Self-Cancellation Scheme for OFDM Mobile Communication Systems," IEEE Trans. Commun., vol. 49, No. 7, July 2001.

[11] H. Yeh, Y. Chang, and B. Hassibi, " A Scheme for Cancelling Intercarrier Interference Using Conjugate Transmission in Multicarrier Communication Systems," IEEE Trans. Wirel. Commun., vol. 6, No. 1, January 2007.

[12] M. Guenach and H. Steendam, "Performance Evaluation and Parameter Optimization of MC-CDMA,” IEEE Trans.Vech. Technol., Vol. 56, No. 3, pp. 1165-1175, May 2007.
[13] L.L. Yang, and L. Hanzo, M. Young, “ Performance of Fractionally

[14] Spread Multicarrier CDMA in AWGN as Well as Slow and Fast

[15] Nakagami- $m$ Fading channels," IEEE Trans.Veh.Technol vol. 54, No. 5, Sept. 2005, pp. 1817-1827.

[16] L.L. Yang and L. Hanzo, "Performance of Generalized Multicarrier DS-CDMA Over Nakagami-m Fading Channels," IEEE Trans. Commun., vol.50, pp. 956-966, June 2002.

[17] R.K. Morrow, "Accurate CDMA BER Calculations with Low Computational Complexity," IEEE Tranl. Commun., vol. 46, No. 11, Nov. 1998, pp. 1413-1417.

[18] A. Papoulis and S. U. Pillai, Probability, Random Variables and Stochastic Processes, $4^{\text {th }}$ ed., pp. 162, pp. 131. New York: McGraw Hill, 2002.

[19] M. K. Simon and M.-S. Alouini, "A unified approach to the Performance analysis of digital communication over generalized fading channels," Proc. IEEE, vol. 86, pp. 1860-1877, Sep.1998.

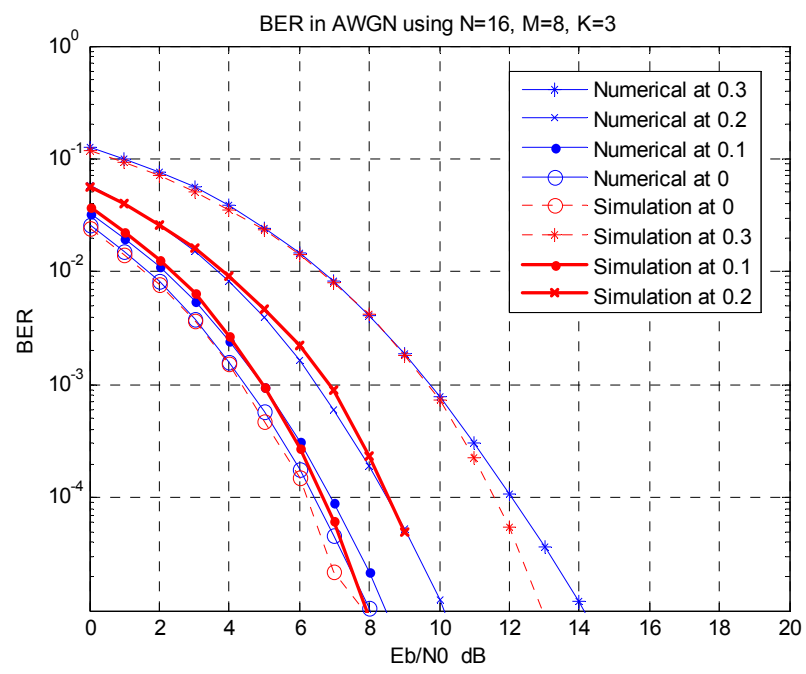

Fig. 4 Simulated BER performance of TF-domain spread MC DS-CDMA, using random spreading sequences in AWGN channel

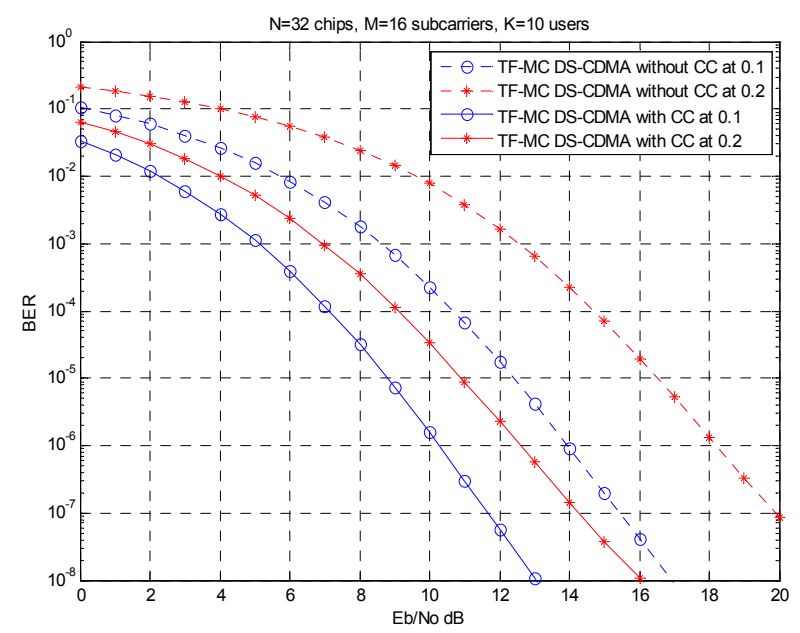

Fig.5 Performance of TF-domain spread MC DS-CDMA, with and without $\mathrm{CC}$ in $\mathrm{AWGN}$ channel at $\varepsilon=0.1$ and 0.2 


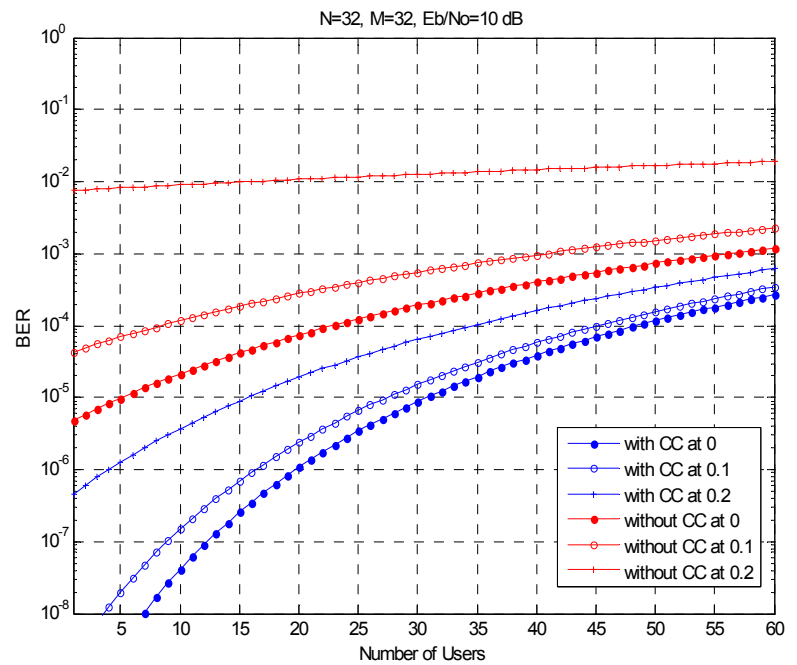

Fig.6 BER performance of TF-domain spread MC DS-CDMA, with and without $\mathrm{CC}$ versus number of users in AWGN channel.

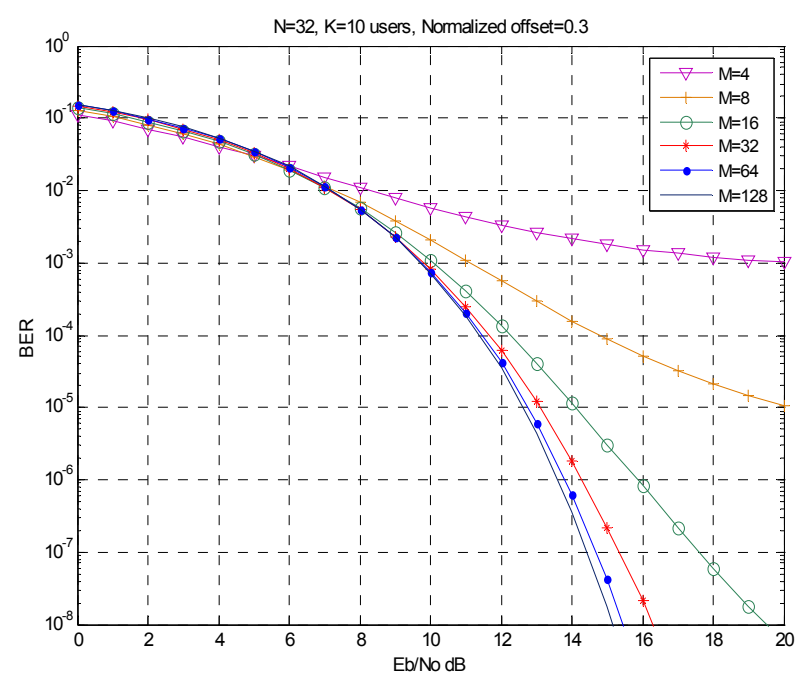

Fig.7 BER performance of TF-domain spread MC DS-CDMA, at different values of subcarriers in AWGN channel

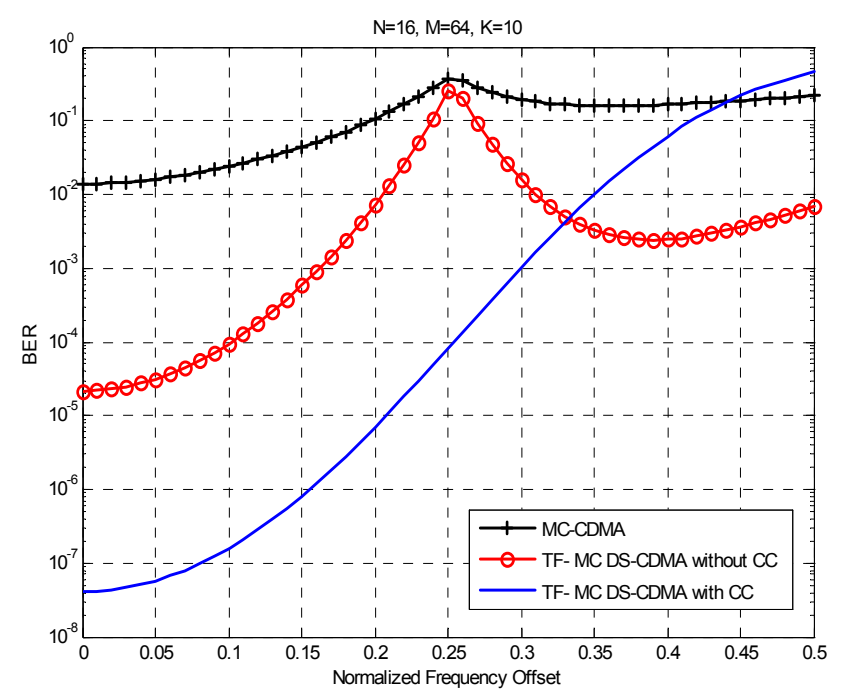

Fig. 8 BER performance of TF- MC DS-CDMA with and without CC, conventional MC-CDMA versus normalized frequency offset in AWGN channel.

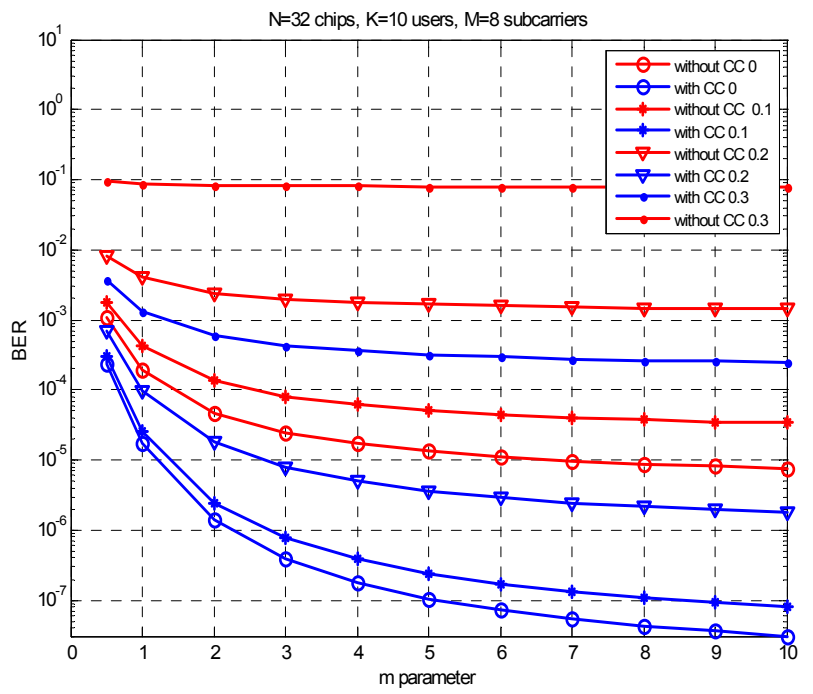

Fig.9 BER performance of TF- MC DS-CDMA with and without $\mathrm{CC}$ versus the Nakagami-m parameter in fading channel 